\title{
The switching control strategy of redundant electromechanical servo system based on the compound sliding mode control
}

\author{
Zonglun $\mathrm{Li}^{*}$, Zhiyuan $\mathrm{Yu}$, and Menglong Jiang \\ Laboratory of Aerospace Servo Actuation and Transmission,Beijing 100076, China
}

Keywords: sliding mode control, redundancy, active-active mode.

\begin{abstract}
Aiming to resolve the limitations of robustness and stability in switching working modes of dual redundancy electric servo system, a control strategy based on sliding mode control is suggested. By analyzing the electromechanical servo system's basic structure and working theory, a mathematical model is created to enable the design of new control functions. Taking into account the influence of the internal parameter perturbation of the electromechanical servo system during the switching between working modes, the sliding mode control is used to enhance system robustness, and the method of combining the latest saturation function and the exponential reaching law is used to minimize the system chattering. The simulation results and analysis show that the control strategy can effectively improve the robustness and control accuracy of the system.
\end{abstract}

\section{Introduction}

In the past decade, redundant technology has become a key technology for the aerospace sector because of its high reliability. Among the solutions identified for the redundancy electromechanical servo system, the dual-motor differential electromechanical servo system has a promising future and important significance[1-2]. Compared with single-channel servos, the dual-channel design consists of two motors which in the active-active working mode can effectively use resources, meanwhile, the redundant design greatly improves the reliability of the system. However, when a fault occurs and the operating mode needs to be switched, the rotary inertia and other parameters of the system change significantly, which requires the system to have fast response performance, better response accuracy, and strong robustness.

Sliding mode control is a device that uses discontinuous control which is different from PID and other existing control strategies[3-4]. With the sliding mode configuration, the device is insensitive to the change in the control parameters and has tremendous robustness. Hence, its use in electronic servo systems is noteworthy. However, sliding mode variable structure control triggers system chattering, and researchers have suggested some solutions:

\footnotetext{
* Corresponding author: 18686800261@163.com
} 
based on a reaching rule method[5-7], boundary layer method[8], terminal sliding mode[9-11], sliding mode-predictive control method[12], sliding mode-fuzzy control[13], high-order sliding mode method[14-16], etc. and how to suppress chattering has long been a research hotspot in related fields.

In this paper, the sliding-mode variable structure control is applied to a dual-motor differential electromechanical servo system to boost responses and facilitate homogeneity. The speed loop developed using the combination of the exponential reaching law, and a new saturation function, which is effective at suppressing chatter. In addition to the traditional PID control technique, an air rudder deflection output and motor speed curve were evaluated and the overshoot was decreased, the response time was quicker, and the system was more robust. The verification of results confirms the validity of the study and its results.

\section{Mathematical model of the electromechanical servo system}

The dual-motor differential electromechanical actuator consists of two permanent magnet synchronous motors, NGW planetary reducer, and a ball screw mechanism. The schematic diagram of the servo system is shown in Figure 1.

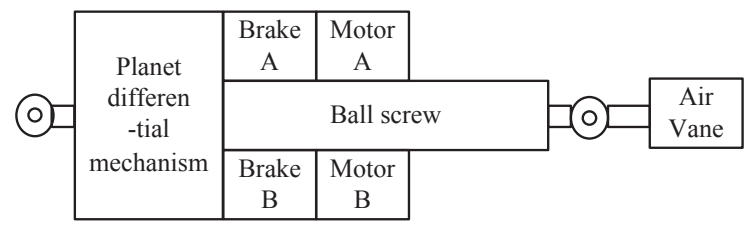

Fig. 1. The schematic diagram of the servo system.

\subsection{Modeling of permanent magnet synchronous motors}

First, hypothesize that a permanent magnet synchronous motor would act as a model for an ideal electric motor.

1) The stator windings are uniform and symmetric with no turns; 2) The air gap magnetic field is a normal sinusoidal distribution; 3 ) The eddy current losses and magnetic saturation in the iron core are ignored; 4) The rotor has no damping effect, and the magnetic connection amplitude is constant[17]. The equation for the magnetic field of the permanent magnet stepper motor is as follows:

For surface-mounted permanent magnet synchronous motors:

$$
\left\{\begin{array}{l}
u_{d}=R \cdot i_{d}+\frac{d \lambda_{d}}{d t}-\omega_{e} \cdot \lambda_{q} \\
u_{q}=R \cdot i_{q}+\frac{d \lambda_{q}}{d t}+\omega_{e} \cdot \lambda_{q}
\end{array}\right.
$$

where $\lambda_{d}=\mathrm{L}_{d} \mathrm{i}_{d}+\psi_{d}, \lambda_{q}=\mathrm{L}_{q} \mathrm{i}_{q}+\psi_{q}$ are the total flux of $\mathrm{d}$ and $\mathrm{q}$ axis respectively; $\psi_{d}, \psi_{q}$ are the flux of $\mathrm{d}$ and $\mathrm{q}$ axis's permanent magnet; $\mathrm{u}_{d}, \mathrm{u}_{q}, \mathrm{i}_{d}, \mathrm{i}_{q}$ are the voltage and current of motor stator respectively; $\mathrm{R}$ is the resistance of stator winding; $\mathrm{L}_{\mathbf{d}}, \mathrm{L}_{\mathbf{q}}$ are the inductance of $\mathrm{d}$ and $\mathrm{q}$ aixs.

The rotor mechanical motion equation of the permanent magnet synchronous motor is as follows: 


$$
J \frac{d \omega_{m}}{d t}=T_{e}-T_{d}-B \cdot \omega_{m}
$$

where $\omega_{m}$ is the mechanical angular velocity; $J$ is the rotary inertia; $T_{e}$ is the electromagnetic torque; $T_{d}$ is the load torque; $B$ is the friction factor.

Permanent magnet synchronous motor rotor electromagnetic torque equation:

$$
T_{e}=\frac{3}{2} p_{n}\left(\lambda_{d} \cdot i_{q}-\lambda_{q} \cdot i_{d}\right)=\frac{3}{2} p_{n}\left(\psi_{d} \cdot i_{q}+\left(L_{d}-L_{q}\right) \cdot i_{d} \cdot i_{q}\right)=K_{t} \cdot i_{q}
$$

where $\mathrm{K}_{t}$ is the motor torque constant; $\mathrm{p}_{n}$ is the logarithmic magnetic pole of the rotor.

\subsection{Modeling of the mechanical transmission mechanism}

The mechanisms of the dual-motor differential electromechanical servo system are composed of motor, NGW reducer, and ball screw. The motor generates torque, and the planetary reducer couples it with the ball screw shaft, then the screw shaft translates the torque into axial force to drive the air rudder load. By modifying the voltage supplied to the motor and using the electromechanical servo system, you can accurately monitor the amount of force and speed needed.

The mathematical model of how a dual-motor differential electromechanical servo device operates gives us the equation for the system.

1) NGW type planetary reducer

To minimize power consumption, the NGW style planetary reduction gear was selected for the double redundancy electromechanical servo system. The arrangement of the reducer is shown in Figure 3. The sun gear and planet carrier are coupled to the A channel, and the ring gear and drive gear are coupled to the B channel.
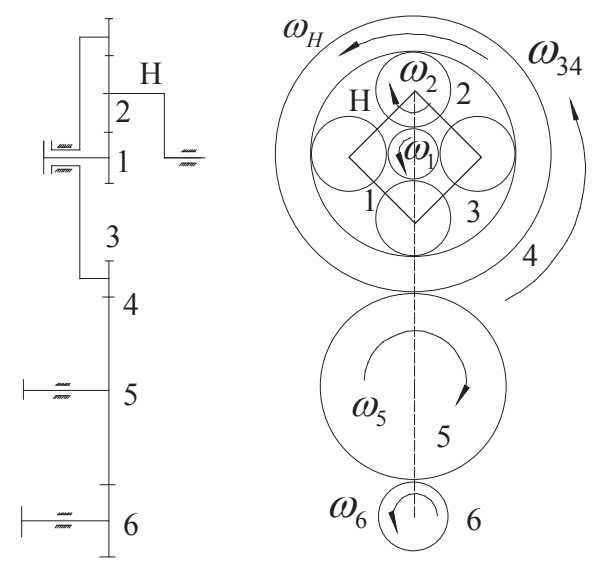

Fig. 2. NGW type planetary reducer.

According to the various working modes of the device, the reducer is divided into three working states: A channel and B channel dual input, A channel single input (sun gear side input), and B channel single input (ring gear side input). The dual input ratio is 2.5 and the single input ratio is 5 . The set consists of $\mathrm{T}_{1}$ and $\mathrm{T}_{6}$, the two input torques, and $\mathrm{T}_{H}$, the load torque. The speed of the reducer is obtained by using the equation:

(1) Two channel dual input: 


$$
T_{H}=-\frac{1}{5} T_{1}-\frac{1}{5} T_{6}+b_{1} \varepsilon_{1}+b_{2} \varepsilon_{2}
$$

(2) One channel single input(ring gear side input):

$$
T_{H}=-\frac{1}{5} T_{6}+b_{24 \varepsilon 6}
$$

(3) One channel single input(sun gear side input)

$$
T_{H}=-\frac{1}{5} T_{1}+b_{23} \varepsilon_{1}
$$

where $\mathrm{b}, \varepsilon$ are the scale coefficient of interia and accelerated speed.

2)

Ball screw model:

$$
m_{b s} \ddot{x}_{e m a}=F_{G}-F_{f}-F_{e m a}
$$

where $\mathrm{m}_{b s}$ is the mass of the ball screw; $\mathrm{F}_{G}$ is the load capacity between ball bearing and screw; $\mathrm{F}_{f}$ is the friction force; $\mathrm{F}_{\text {ema }}$ is the external force of the EMA.

$$
F_{G}=T_{G} i_{t r} i_{b s}=T_{G} i_{t r} \frac{2 \pi}{p_{b s}}
$$

where $\mathrm{T}_{G}$ is output torque of motor; $\mathrm{i}_{t r} \mathrm{i}_{b s}$ are reduction ratio of reducer and ball screw; $\mathrm{p}_{b s}$ is the helical pitch of ball screw.

Assuming that all collisions of the electromechanical actuator are completed in an instant, the dead zone theory is introduced to simulate the existence of the gap in the hinge of the actuator. The $\mathrm{x}_{0}$ represents the total gap while the $\mathrm{x}_{m}, \mathrm{x}_{l}$ are displacements of the ball screw driven by motor and load.

$$
F_{G}=\left\{\begin{array}{cc}
k_{s} \cdot\left(x_{m}-x_{l}-x_{0}\right) & x_{m}-x_{l}>x_{0} \\
0 & \left|x_{m}-x_{l}\right|=x_{0} \\
k_{s}\left(x_{m}-x_{l}+x_{0}\right) & x_{m}-x_{l}<-x_{0}
\end{array}\right.
$$

3)

$$
\text { Air vane model }
$$

$$
m_{c s} \ddot{x}_{c s}=F_{e m a}-F_{a i r}=c_{c s}\left(x_{e m a}-x_{c s}\right)+d_{c s}\left(\dot{x}_{e m a-} \dot{x}_{c s}\right)-F_{a i r}
$$

where $\mathrm{m}_{c s}, \mathrm{x}_{c s}$ are the mass and displacement of the air vane; $\mathrm{F}_{\text {air }}$ is the aerodynamic load acting on the rudder surface; $\mathrm{c}_{c s}, \mathrm{~d}_{c s}$ are stiffness coefficient and viscous damping coefficient of the air vane. 


\section{Speed loop sliding mode controller design}

Traditional PI control is sensitive to parameter changes and has a poor dynamic response, which cannot meet the requirements of high-performance servo systems. Sliding mode control is a variable structure control[22]. Due to its strong robustness and irrelevance to system parameter changes, it has become a research hotspot in motor control controller design in recent years. This paper focuses on the stability issue of the dual-motor differential electromechanical servo system during the switching of operating modes, and thus introduces speed loop sliding mode control using PID three-loop control. However, sliding mode control also brings in the uncooperative condition of unpredictable noise production. The sliding mode controller is replaced with an arctangent feature, reducing the chattering caused by the controller's motion.

The concept and implementation of a sliding mode controller for exponentially rising speed are as follows.

$$
e=x^{\prime}{ }^{\prime}-x^{\prime}
$$

As the actual current $\mathrm{i}_{q}=\mathrm{x}$, , set the predicted current value $\mathrm{i}_{q d}=\mathrm{x}_{d}{ }_{d}$, then the error equation is:

$$
\dot{e}=\dot{x}^{\prime}{ }_{d}-\dot{x}^{\prime}
$$

Designing of sliding mode surface:

$$
s=e+c \cdot \int e d t
$$

Set $c>0, \varepsilon$ is the constant-velocity reaching law coefficient, $\mathrm{k}$ is exponential reaching law coefficient, the design of reaching law is given by:

$$
\dot{s}=-\varepsilon \cdot \operatorname{sign}(s)-k \cdot s
$$

Combine equation(2)and the derivation of equantion(14):

$$
\dot{e}=\dot{\omega}_{r e f}-\dot{\omega}_{m}=\dot{\omega}_{r e f}-\left(\frac{K_{t}}{J} i_{q}-\frac{T_{L}}{J}-\frac{B}{J} \omega_{m}\right)
$$

Refers to equation(14) we can get the output of the speed-loop controller:

$$
i_{q}^{*}=\frac{J}{K_{t}}\left[\omega_{r e f}+\frac{T_{L}}{J}+\frac{B}{J} \omega_{m}+c \cdot e+\varepsilon \cdot \operatorname{sign}(s)+k \cdot s\right]
$$

Via mathematical analysis, the increase of $\mathrm{c}$ and $\mathrm{k}$ will boost the response of the control system and minimize the tracking error, and the decrease of $\varepsilon$ will slow down the chattering, but it will also cause the system to slow down the tracking speed. $\varepsilon \cdot \operatorname{sign}(\mathrm{s})$ is the item that causes the chattering of the control quantity. The existence of this switching function is the key to the advantage that it can ignore the change of the control parameter. Many researchers are trying to design tons of sat(s) to replace the sign(s)[18-21]. In this paper, the arctangent function: $\operatorname{sat}(\mathrm{s})=\arctan \left(\mathrm{c}_{0} \cdot \mathrm{s}\right)$ is used in place of the sign function because of its simpler properties and fewer parameter choices. The arctangent function curve is smoother and more distinct, and the essence of reducing chattering is that multiplying with $\varepsilon$ changes the benefit of the constant velocity approaching law. When the value of sliding mode 
surface $s$ is large, the system gain is a large setting value When approaching the sliding mode surface, the system gain decreases as the saturation function value becomes smaller. This design not only retains the sliding rate close to the sliding mode surface but also reduces the fluctuation of the control amount of the sliding phase. The structure is simple which facilitates the development of the engineering design.

Define the Lyapunov stability equation

$$
V=\frac{1}{2} s^{2}
$$

The derivation of equation(17) is:

$$
\dot{V}=s \cdot \dot{s}=s\left(-\varepsilon \cdot \arctan \left(c_{0} \cdot s\right)-k \cdot s\right)=-s \cdot \varepsilon \cdot \arctan \left(c_{0} \cdot s\right)-k \cdot s^{2}
$$

where $\arctan$ function is odd, when $k>0, c_{0}>0, \varepsilon>0, \dot{V}=s \cdot \dot{s}<0$,

So the control law is progressively stable in the Lyapunov sense, which is global convergence.

Finally, we can obtain the output of the sliding mode controller is:

$$
i_{q}^{*}=\frac{J}{K_{t}}\left[\omega_{r e f}+\frac{T_{L}}{J}+\frac{B}{J} \omega_{m}+c \cdot e+\varepsilon \cdot \arctan \left(c_{0} \cdot s\right)+k \cdot s\right]
$$

\section{Simulation results and analysis}

By establishing a dual-motor differential electromechanical servo system model in MATLAB/SIMULINK, as shown in Figure 3. The values of some of the simulation's vector parameters are shown in Table 1 .

Table 1. Value of variable in the simulation model.

\begin{tabular}{c|c|c}
\hline No. & Variable & Value \\
\hline 1 & $L_{m}$ & $0.0018 \mathrm{H}$ \\
\hline 2 & $R_{m}$ & $0.15 \Omega$ \\
\hline 3 & $K_{t}$ & $0.37 \mathrm{Nm} / \mathrm{A}$ \\
\hline 4 & $B_{A}=B_{B}$ & 0.004 \\
\hline 5 & $K_{A}=K_{B}$ & $3 \times 10^{5} \mathrm{Nm} / \mathrm{rad}$ \\
\hline 9 & $B_{L}$ & 0.005 \\
\hline 10 & $K_{L}$ & $2.54 \times 10^{7} \mathrm{~N} / \mathrm{m}$ \\
\hline 11 & $T_{f}$ & $4000 \mathrm{~N} \cdot \mathrm{m}$ \\
\hline
\end{tabular}




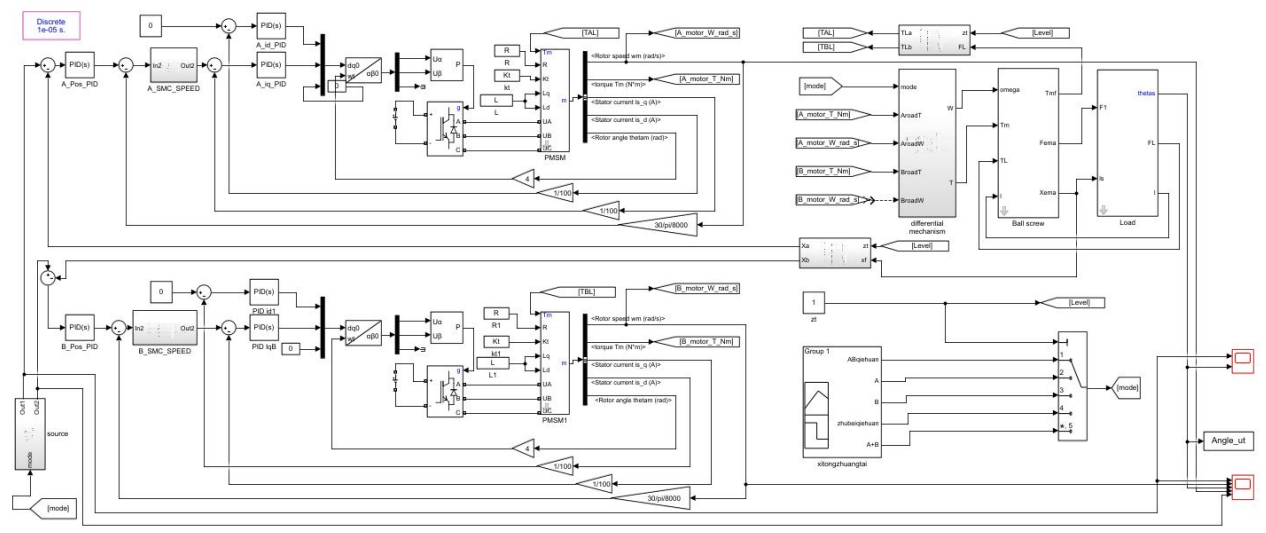

Fig. 3. electromechanical servo system model.

\subsection{Application of arctangent function.}

Based on the above simulation model, a single-channel mode is adopted, the speed loop sliding mode controller is selected, the step response is input, and the simulation verification is performed. The speed curve using the switching function is shown in the figure, and the speed curve using the arctangent function is shown in figure 4.

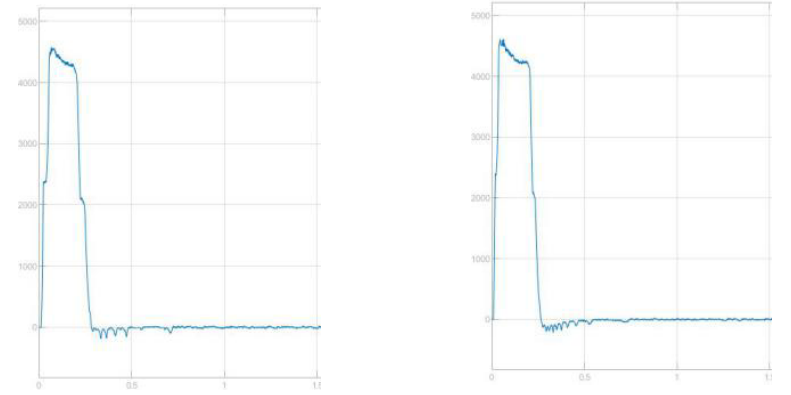

Fig. 4. Sign function's speed curve (left)and the arctangent function's speed curve(right).

From Figures 4 and 5, it can be seen intuitively that the speed fluctuation in the output of the sliding mode controller by the traditional exponential sliders is greater than the speed fluctuation in the output of the improved sliders. When the system reaches the vicinity of the sliding mode surface When near the sliding mode surface, the law of reaching the sign function is the law of reaching the switching function, and the value of the s function shifts rapidly, passing back and forth near the critical point of the s function. The controller of the new design type can smoothly reduce the approach gain due to the multiplication of the original constant approach rate with the smoothly decreasing arctangent function at the time when the sliding mode surface is about to be reached. The use of the arctangent function instead of the switching function is simple in structure, easy to adjust parameters, and can reduce system chattering to a certain extent.

\subsection{Working mode switching}

Aiming at the different working modes of the dual-motor differential electromechanical servo system, this paper simulates the active-standby model switching to the active-standby mode 2 (B channel $\rightarrow$ A channel) and the active-active mode switching to 
the active-standby mode $(\mathrm{A}+\mathrm{B}$ channel $\rightarrow 2 \mathrm{~A}$ channel $)$. The input adopts a sine curve, and the two algorithms of conventional PID three-loop control and speed-loop sliding mode control are respectively used. The simulation models switches at the zero position of the sine curve(2s), at which time the speed is maximum.to obtain the angle of the air vane and the motor speed curve as follows shown.

Figure 5 shows the active-active mode switching to the active-standby mode $(\mathrm{A}+\mathrm{B}$ channel $\rightarrow 2 \mathrm{~A}$ channel) Figure6 shows the active-active mode switching to the active-standby mode(B channel $\rightarrow$ A channel)

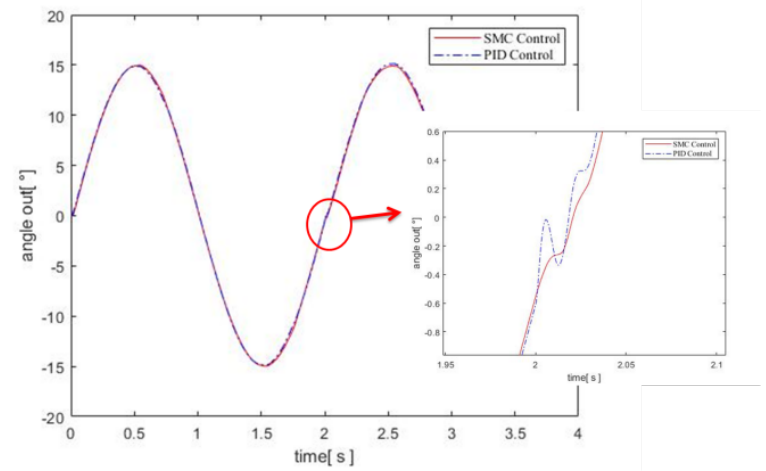

(a)The angle of air vane
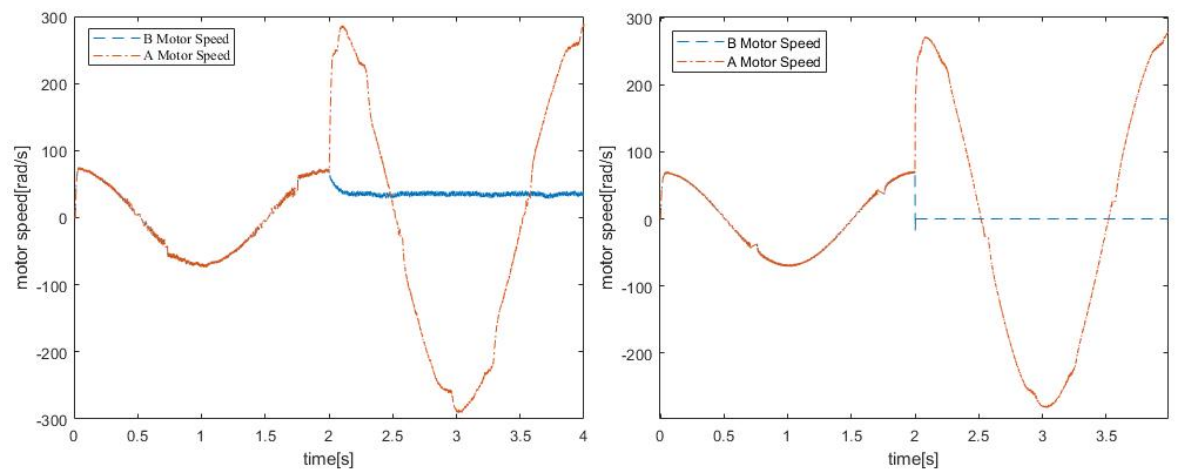

(b) PID control's speed curve (left) SMC control's speed curve(right)

Fig. 5. (A+B channel $\rightarrow 2 A$ channel).

By observing figure 5, it is clear to know that although the system's chatting of the PID control is not very large, but the SMC control's system curve is much smoother, comparing the speed curve, the B channel under the PID control can not return to zero due to the static error, and the SMC control is the better algorithm. 


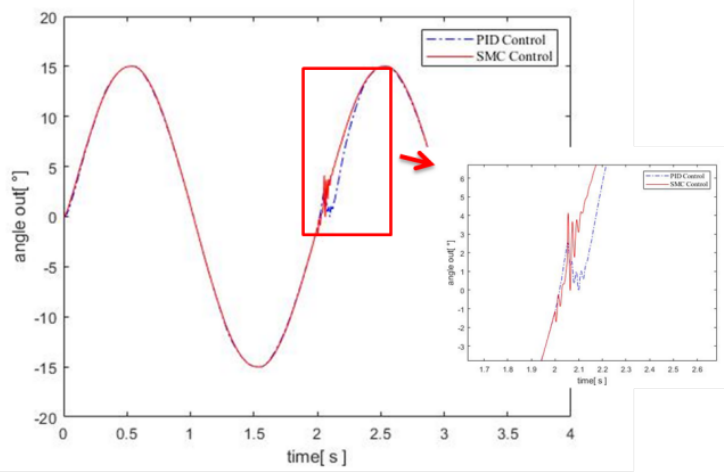

(a)The angle of air vane
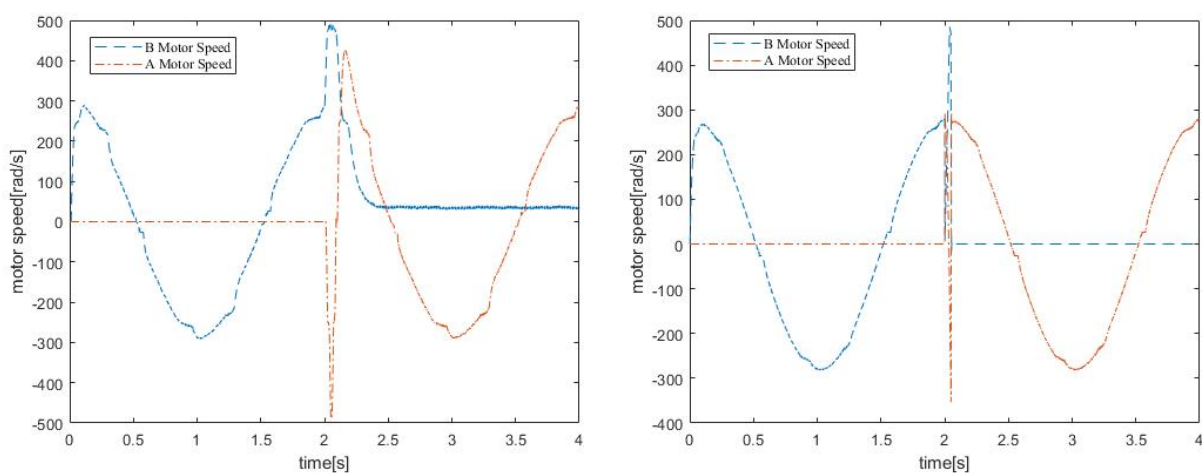

(b) PID control's speed curve (left) SMC control's speed curve(right)

Fig. 6. (B channel $\rightarrow$ A channel).

Looking at Figures 5 and 6, sliding mode control greatly improves the control accuracy of the system. The sliding mode control has a smaller overshoot and a quicker answer than the PID control. As shown in Figure 6, when B channel $\rightarrow$ A channel, the switching time between speed is about $0.15 \mathrm{~s}$, and the speed overshoot is $29.3 \%$. However, when the $\mathrm{A}+\mathrm{B}$ channel $\rightarrow 2 \mathrm{~A}$ channel, the switching time between speed is about $0.1 \mathrm{~s}$, and the speed overshoot is $5 \%$.

It can be seen that, compared with the active-standby mode1 switching to the active-standby mode 2, the overshoot of the active-active mode switching to the active-standby mode is smaller and the switching time is shorter. The active-active working mode is better.

\section{Summary}

This paper builds a model of a dual-motor differential electromechanical servo system that takes into account inertia, friction load, and clearance. Aiming at the problems of low stability and poor control accuracy during the process of working mode switching. A new sliding mode control algorithm is proposed by combining the arctangent function and the power-law function. Simulation experimental results show that the speed loop sliding mode control algorithm eliminates speed fluctuations and suppresses controller chatter, increases robustness, and uses the arctangent function instead of the switching function. 


\section{References}

1. D. Arriola and F. Thielecke: Design of fault-tolerant control functions for a primary flight control system with electromechanical actuators,2015 IEEE AUTOTESTCON, National Harbor, MD, 2015, pp. 393-402

2. David Arriola, Frank Thielecke: Model-based design and experimental verification of a monitoring concept for an active-active electromechanical aileron actuation system, Mechanical Systems, and Signal Processing, vol. 94, pp. 322, 2017.

3. Liu Jinkun, Sun Fuchun. Research on Theory and Algorithm of Sliding Mode Variable Structure Control And progress [J] . Control Theory and Application, 2007, 24(3): 407-418.

4. YE Chao, LI Hongwen.PMSM Sliding Mode Control Based on Novel Saturation Function and PI Observer, Journal of Chongqing Institute of Technology,2020, 34(5).

5. Y. Li and L. Liu: The research of the sliding mode control method based on improved double reaching law,2018 Chinese Control And Decision Conference (CCDC), Shenyang, 2018, pp. 672-675.

6. Q. Hu, L. Liu, and H. Yang: A sliding mode speed controller based on novel reaching law of permanent magnet synchronous motor system, 2017 Chinese Automation Congress (CAC), Jinan, 2017, pp. 954-958.

7. A. K. Junejo, W. Xu, C. Mu, M. M. Ismail, and Y. Liu, "Adaptive Speed Control of PMSM Drive System Based a New Sliding-Mode Reaching Law," in IEEE Transactions on Power Electronics, vol. 35, no. 11, pp. 12110-12121, Nov. 2020.

8. Cui Yalong, Yang Yonghao.Research on an Adaptive Fuzzy Sliding Mode Boundary Layer Control Method for a Hypersonic Vehicle, Computer Measurement \& Control,2014, 22(5):1426-1429,1432

9. Z. Qin, X. He, and D. Zhang, "Nonsingular and fast convergent terminal sliding mode control of robotic manipulators," Proceedings of the 30th Chinese Control Conference, Yantai, 2011, pp. 2606-2611.

10. Zengcheng ZHOU, Guoyuan TANG, Hui HUANG, et al.Adaptive nonsingular fast terminal sliding mode control for underwater manipulator robotics with asymmetric saturation actuators[J].Control Theory and Technology,2020,18(1):81-91.

11. Aravind M.A., Dinesh N.S., K. Rajanna Application of EMPC for precise position control of DC-motor system with Backlash[J] Control Engineering Practice, 2020, 100.

12. Jianguo Zhou, Zhiyuan Liu, and Run Pei, "Sliding mode model predictive control with terminal constraints," Proceedings of the 3rd World Congress on Intelligent Control and Automation (Cat. No.00EX393), Hefei, 2000, pp. 2791-2795 vol.4.

13. T. Kaletsanos, S. H. Xepapas, and S. N. Manias, "A novel sliding mode fuzzy logic control technique for induction motor drive systems," 2001 IEEE 32nd Annual Power Electronics Specialists Conference (IEEE Cat. No.01CH37230), Vancouver, BC, 2001, pp. 1209-1214 vol.2.

14. D. Chen, H. Du and X. Jin, "Position tracking control for permanent magnet synchronous motor based on integral high-order terminal sliding mode control," 2017 32nd Youth Academic Annual Conference of Chinese Association of Automation (YAC), Hefei, 2017, pp. 234-239

15. Brahim BRAHMI, Mark DRISCOLL, Mohamed Hamza LARAKI, et al.Adaptive high-order sliding mode control based on quasi-time delay estimation for uncertain robot manipulator[J].Control Theory and Technology,2020,18(3):279-292. 
16. Djemai, M., Busawon, K., Benmansour, K., et al.High-order sliding mode control of a DC motor drive via a switched controlled multi-cellular converter[J]. International Journal of Systems Science: The Theory and Practice of Mathematical Modelling, Simulation, Optimization and Control in Relation to Biological, Economic, Industrial, and Transportation Systems,2011,42(10/12):1869-18 82.

17. FALLAHA C J, SAAD M, KANAAN H Y, et al. Slidingmode robot control with exponential reaching law $[\mathrm{J}]$. IEEE Transactions on Industrial Electronics, 2011, 58 (2): $600-610$.

18. LUENBERGER D. An introduction to observers $[\mathrm{J}]$. IEEE Transactions on Automatic Control, 1971, 16 (6): 596 - 602.

19. He Yikang. Pulse width modulated(PWM) inverterasynchronous motor system steady-state space analysis[J]. Transactions of China Electrotechnical Society, 1994, 9(2): 11-15.

20. Olaf Cochoy, Susan Hanke, Udo B Carl. Concepts for position and load control for hybrid actuation in primary flight controls[J]. Aerospace Science and Technology, 2007, 11(2/3): 194-201.

21. GUO Honggen, WANG Zhiguo. Overview of the Development of Medium and High Power Space Electric Servo Mechanism [J].Navigation Positioning and Timing, 2016, (3): $1-5$.

22. ZHANG Lisong, HU Youde, XU Lixin. Principle and Design of Servo System [M] .Beijing: Beijing University of Technology Press, 2008. 